\title{
Influence de la géométrie de l'environmement aval de la roue sur les caractéristiques des pompes à débit partiel
}

\author{
Influence of the downstream geometrical layout of \\ the impeller on the characteristics of partial flow pumps
}

\author{
G. Caignaert
}

Laboratoire de Mécanique, E.N.S.A.M. Lille

\section{Notations}

$H M$ Hauteur nette au point de rendement maximal;

$Q M$ Débit volume au point de rendement maximal;

$P M$ Puissance sur l'arbre au point de rendement maximal ;

$H 25$ Hauteur nette pour $Q 25=Q M / 4$;

$P 25$ Puissance sur l'arbre pour $Q 25=Q M / 4$;

$H 0$ Hauteur nette à débit nul ;

$P 0$ Puissance sur l'arbre à débit nul ;

$H_{*} \quad$ Hauteur nette maximale;

$P_{*} \quad$ Puissance sur l'arbre au point de hauteur nette maximale ;

$Q_{*} \quad$ Débit volume au point de hauteur nette maximale;

$R_{2} \quad$ Rayon en sortie de roue;

$b_{2} \quad$ Largeur en sortie de roue ;

$R_{3} \quad$ Rayon à l'entrée du diffuseur ou au bec de volute ;

$b_{3} \quad$ Largeur à l'entrée du diffuseur ou de la volute;

$\omega$ Vitesse angulaire de la machine;

$u_{2} \quad u_{2}=\omega \cdot R_{2}$;

$C_{2} \quad$ Vitesse absolue du fluide en sortie de roue;

$C_{u 2}$ Composante circonférencielle de $C_{2}$;

$\alpha_{2 *} \quad$ Angle de $C_{2}$ avec la direction circonférencielle ;

$r \quad$ Rayon du bec de volute ou à l'entrée du profil du diffuseur ;

$\alpha_{3} \quad$ Angle du squelette du profil du bec de volute ou du diffuseur avec la direction circonférencielle;

$a_{3} \quad$ Col du diffuseur (Fig. 9);

$Z_{\mathrm{d}} \quad$ Nombre d'aubages du diffuseur;

$S_{9} \quad$ Section en sortie de volute (Fig. 2);

$\Delta \quad$ Jeu radial relatif $\Delta=\left(R_{3}-R_{2}\right) / R_{2}$;

$\eta_{h} \quad$ Rendement hydraulique;

$\psi \quad$ Coefficient de hauteur $\psi=2{ }_{\eta_{h}} C_{u_{2}} / U_{2}$; $\phi \quad$ Coefficient de débit $\phi=Q /\left(2 \pi R_{2} b_{2} u_{2}\right)$;

$\Lambda \quad$ Coefficient de rayon $\Lambda=R_{2} \cdot(g H M)^{1 / 4} / Q M^{1 / 2}$;

$\Omega \quad$ Coefficient de vitesse angulaire

$$
\Omega=\omega Q M^{1 / 2} /(g H M)^{3 / 4} .
$$

\section{Introduction}

De nombreux auteurs, tels Knapp [1] et Rütschi $[2,3,4]$, ont depuis longtemps noté l'importance des paramètres géométriques de l'environnement de la roue d'une turbomachine sur ses caractéristiques de fonctionnement. Lorsqu'on aborde le comportement à débit partiel des turbomachines, il apparait que les organes d'aspiration et de récupération d'énergie cinétique jouent un rôle important au même titre que la roue.

Notre étude est consacrée à l'influence des paramètres géométriques des parties situées à l'aval de la roue sur les caractéristiques des pompes à débit partiel. A cet effet, nous avons utilisé des documents internes à certains constructeurs des sous-groupes de la Société Hydrotechnique de France et des documents publiés, relatifs à des essais de turbomachines, (pompes et ventilateurs), pour lesquels des modifications géométriques ont été effectuées, à l'aval de la roue uniquement. Ces résultats ont été mis sous forme de variations des rapports sans dimension $H 0 / H M, P 0 / P M, H 25 / H M$, $P 25 / P M, H_{*} / H M, P_{*} / P M$ (voir notations) en fonction des modifications réalisées. Ces modifications font en général varier simultanément de nombreux paramètres géométriques, et il n'est pas toujours facile de caractériser tous ces résultats autrement que par des tendances. Dans le cadre de cette communication, nous nous sommes limités à la présentation d'un certain nombre de résultats caractéristiques relatifs à des machines centrifuges à volute puis à diffuseur aubé. 
De façon générale, on peut penser que l'influence de l'organe de récupération d'énergie cinétique a d'autant plus d'importance que la fraction d'énergie cinétique récupérable est grande; si on compare l'énergie cinétique massique en sortie de roue $\frac{1}{2} C_{2}^{2}$ au travail massique indiqué $g H_{i}=u_{2} C_{u_{2}}$, on peut écrire :

$$
\frac{C_{2}^{2}}{2 g H_{i}}=\frac{1}{2}\left[\left(\frac{R_{2}}{2 \pi b_{2}}\right)^{2} \cdot \Lambda^{-4} \cdot \eta_{h}+\frac{\Omega^{-2} \cdot \Lambda^{-2}}{\eta_{h}}\right]
$$

ou encore :

$$
\frac{C_{2}^{2}}{2 g H_{i}}=\frac{1}{2}\left[\phi \operatorname{tg} \alpha_{2 *}+\psi / 2 \eta_{h}\right]
$$

Le tracé de ce rapport en fonction de $\Omega$ (Fig. 1), en utilisant les corrélations $\Lambda(\Omega), R_{2} / b_{2}(\Omega)$ et $\eta_{h}(\Omega)$ obtenues dans les ouvrages de Csanady [5] et Sabersky, Acosta et Hauptmann [6] et confirmées par les statistiques effectuées au sein du sous-groupe concerné de la SHF, montre une chute brutale au-delà d'une valeur de $\Omega$ de l'ordre de 1 ; le point de rendement maximal est donc déterminé par les caractéristiques de la volute ou du diffuseur pour les petites valeurs de $\Omega$ et par les pertes dans la roue pour les machines de grand $\Omega$.

De même, il est raisonnable de penser que les perturbations introduites par l'organe de récupération dépendent beaucoup de la plus ou moins grande uniformité de l'écoulement en grandeur et en direction à l'entrée de cet organe tant en azimut qu'axialement. A cet effet, les travaux de Senoo [7] et de Worster [8] donnent des indications utiles sur ce problème de l'uniformisation des vitesses en sortie d'une roue centrifuge.

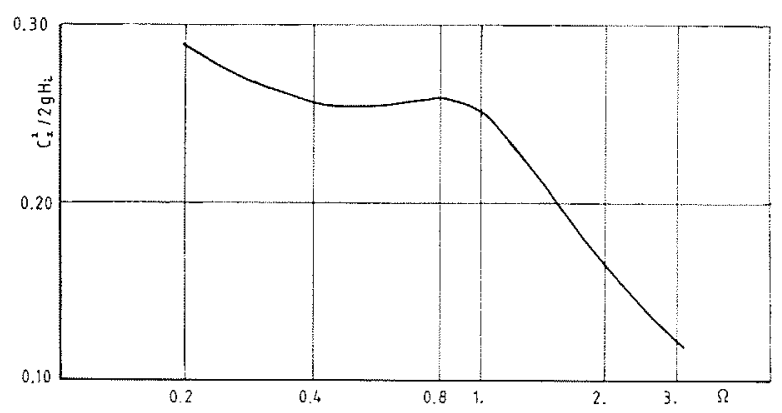

Figure 1 - Récupération d'énergie cinétique.

\section{Pompes centrifuges à simple volute}

\section{Paramètres géométriques}

La figure 2 met en évidence un certain nombre de paramètres géométriques caractéristiques de la volute, et plus particulièrement du bec de volute, partie la plus proche de la roue, qui va donc provoquer des interactions plus ou moins importantes. Dans la mesure du possible, nous avons essayé de préciser les variations relatives de ces paramètres lors de l'analyse des résultats expérimentaux étudiés.

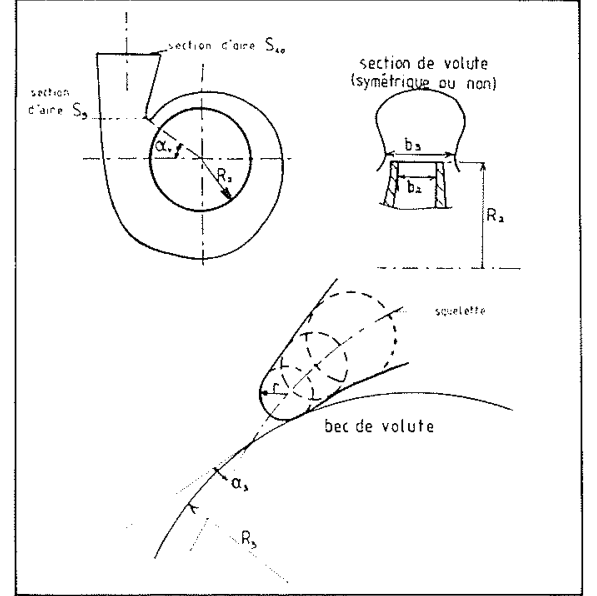

Figure 2 - Paramètres géométriques d'une volute.

\section{Essais de Worster $([9,10])$}

La figure 3 résume un certain nombre de résultats obtenus par Worster [9] avec une même roue placée dans une volute à section rectangulaire $A$, puis dans une volute à section trapézo ïdale $B$, avec un certain nombre de modifications au niveau du bec de volute. La comparaison entre les deux types de volute est délicate car de nombreux paramètres varient simultanément, en particulier la largeur, les sections, les caractéristiques du bec. La comparaison des divers becs $(B 1 \rightarrow B 5)$ montrent les possibilités intéressantes offertes par une modification au niveau du bec de volute pour changer l'allure de la caractéristique de la machine; notons cependant que certains paramètres $\left(\alpha_{3}, r\right)$ ne sont pas connus, ce qui rend difficile l'interprétation. En particulier, la comparaison des configurations $B 3$ et $B 5$ (même jeu relatif $\Delta$ ) semble mettre en évidence l'influence d'une variation de la section $S_{9}$, mais d'autres paramètres ont dû varier simultanément.

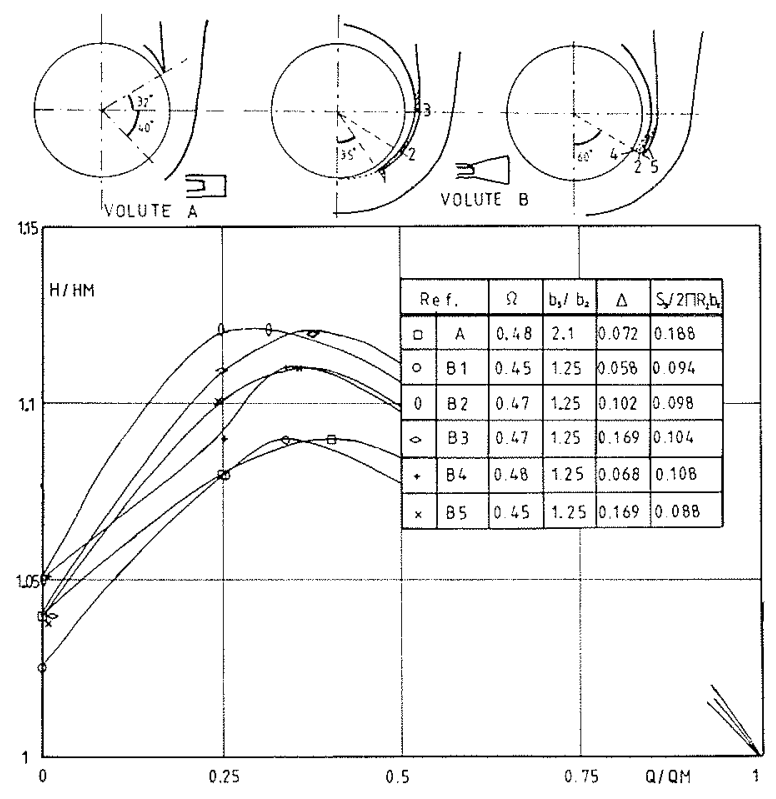

Figure 3 - Essais de Worster [9]. 
La figure 4 montre l'effet d'une rotation du bec; une telle opération modifie non seulement le jeu relatif au niveau du bec, mais également la section $S_{9}$ et l'angle $\alpha_{3}$. On constate cependant l'intérêt d'une rotation du bec dans le sens d'une augmentation du jeu relatif $\Delta$ (et donc d'une diminution de $S_{9}$ ); en effet cette rotation se traduit par une amélioration de $H / H M$ à débit partiel et par une stabilisation de la caractéristique de la machine.

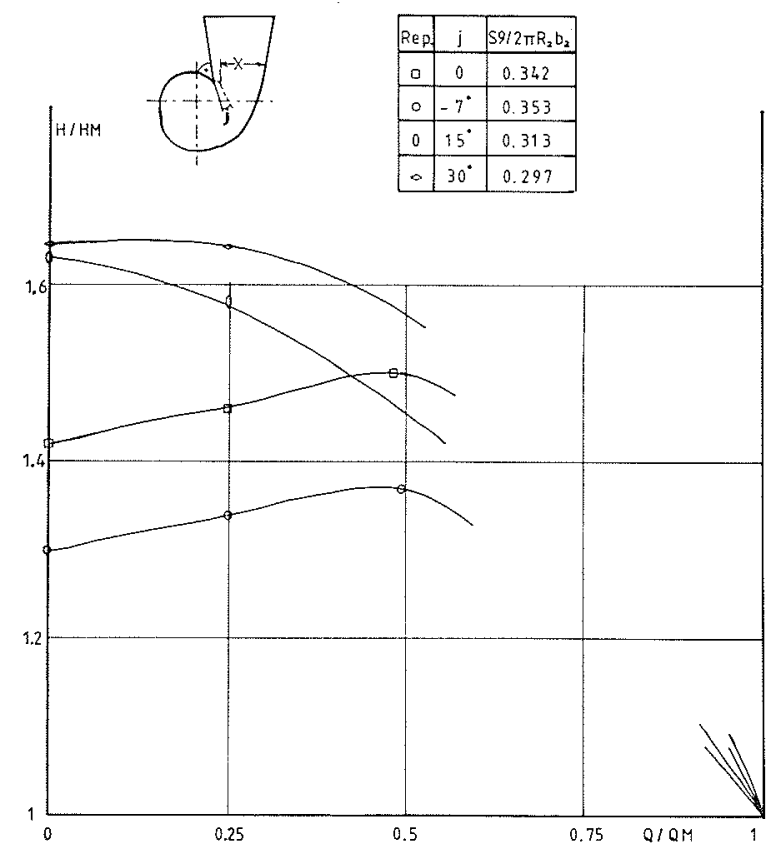

Figure 4 - Essais de Copley, Worster et Zanker [10].

\section{Essais de Kermarec et Dressayre ([11])}

Les figures $5 a$ et $5 b$ montrent respectivement les caractéristiques de volutes essayées au CETIM (Nantes) et les performances obtenues dans diverses configurations.

La comparaison des essais $1 A, 2 A, 3 A$ (ou $1 E, 2 E$, $3 E$ ) permet de mettre en évidence l'influence du tracé général de la volute (choix d'un type de section). On obtient les rapports $H / H M$ les plus grands avec la volute de type 2, qui se caractérise essentiellement par une largeur relative $b_{3} / b_{2}$ plus faible; en ce qui concerne les rapports $P / P M$, on obtient des valeurs plus faibles avec la volute du type 3 , mais ces rapports demeurent proches de ceux obtenus avec le type 2 .

La comparaison des becs $A, E$ et $B$ met surtout en évidence l'influence d'une augmentation du rayon $r$ au bec : lorsque $r$ augmente, les rapports $H / H M$ croissent tandis que les rapports $P / P M$ diminuent.

La comparaison des becs $A$ et $E$ montre surtout les conséquences d'une augmentation du jeu radial entre le bec de volute et la roue : il semble ici qu'une augmentation de ce jeu entraine une diminution des rapports $H / H M$ et une augmentation des rapports $P / P M$.

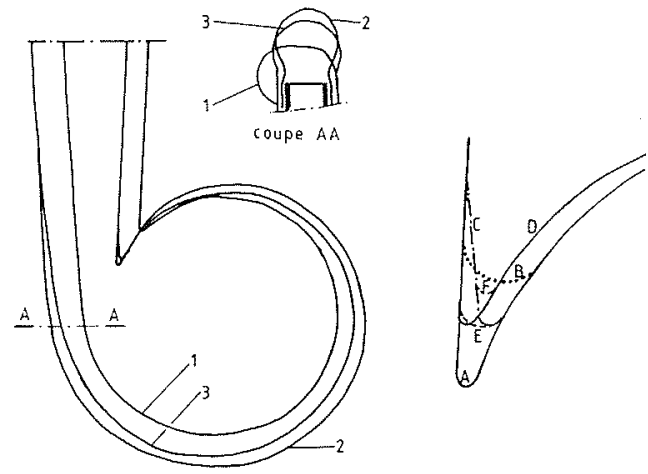

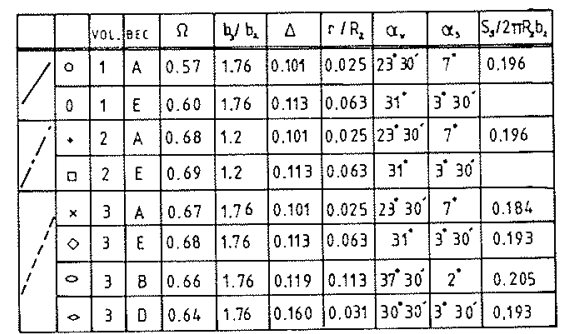

Figure $5 a$ - Caractéristiques géométriques des volutes essayées au CETIM [11].

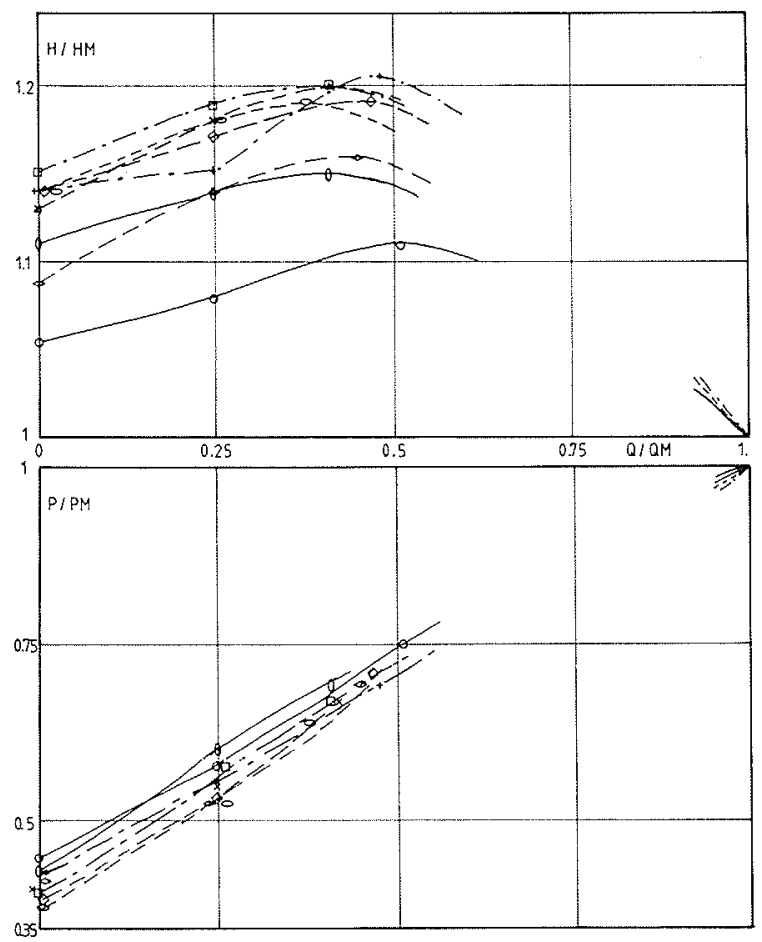

Figure $5 b$-Courbes caractéristiques à débit partiel : essais CETIM [11].

\section{Essais de Suzuki et Ugai ([12])}

Les résultats obtenus pour des ventilateurs centrifuges par Suzuki et Ugai sont résumés par la figure 6 .

Une première série d'essais (roue $A$ ) met en évidence l'effet d'une variation du jeu relatif radial $\Delta$ au bec de volute, en conservant $S_{9}$ et $r$ constants. Notons toutefois que dans une telle opération, l'angle $\alpha_{3}$ évolue 
avec $\Delta$. Une augmentation importante du jeu radial se traduit ici par une augmentation des rapports $H / H M$, entraînant même une tendance à la stabilisation de la caractéristique.

La deuxième série d'essais (roue $B$ ) montre l'influence d'une augmentation du rayon $r$ du bec tout en maintenant constants $S_{9}$ et $\Delta$ : comme précédemment, l'augmentation de $r$ s'accompagne d'un accroissement important des rapports $H / H M$ à débit partiel, avec une tendance à la suppression de la bosse de la caractéristique.

D'autres essais, non représentés ici, indiquent le peu d'influence sur les caractéristiques d'une variation du jeu au bec dans la direction axiale et d'un décalage du bec en azimut.

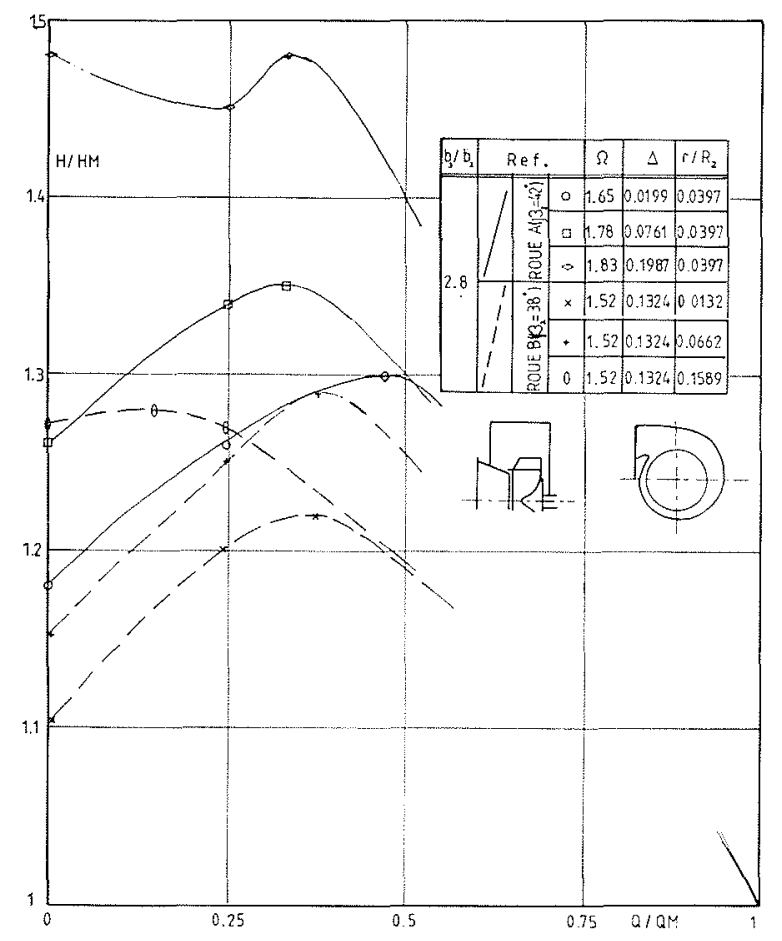

Figure 6 - Essais de Suzuki et Ugai [12].

\section{Essais de Flörkemeier ([13])}

La comparaison de $F 1$ et $F 2$ (Fig. 7) montre l'influence d'une augmentation du jeu au bec : cette augmentation s'accompagne d'une diminution des rapports $H / H M$.

Lorsqu'on passe de $F 5$ à $F 6$, on augmente le rayon au bec, et cela entraîne une augmentation des rapports $H / H M$. L'importance de l'arrondi au bec est mis en évidence par la comparaison de $F 3$ (bec arrondi) et de F4 (bec coupé).

L'observation des caractéristiques $F 1, F 2, F 3, F 5$ et $F 6$ montre bien l'importance des paramètres $r$ et $\Delta$ et la nécessité de réaliser un compromis dans le choix de ces paramètres.

La comparaison de $F 7$ et $F 8$ indique la nécessité d'avoir un canal de sortie peu divergent pour obtenir de bonnes caractéristiques $H / H M$. Enfin, l'analyse des essais $F 9, F 10$, et $F 11$, dont les courbes correspon- dantes n'ont pas été tracées, pour ne pas surcharger la figure 7, montre l'importance du positionnement angulaire du bec dans le cas d'une pompe à sortie radiale.

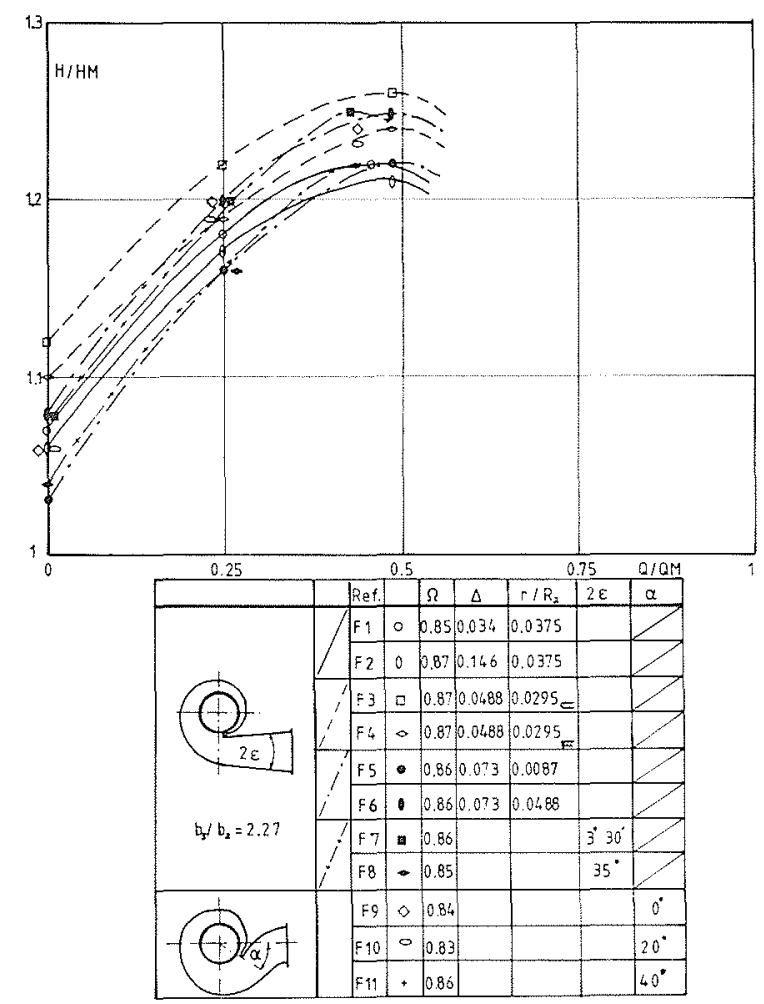

Figure 7 - Essais de Flörkemeier [13].

\section{Exemple de rognages du bec (Fig. 8)}

L'exemple, proposé par un constructeur de pompes, permet de retrouver un certain nombre des conclusions des essais précédents.

La comparaison des becs $C$ et $D$ montre l'intérêt d'une augmentation du rayon $r$ du bec (sans augmenter le jeu radial) pour améliorer les caractéristiques à débit partiel au niveau des puissances $P / P M$.

La comparaison des becs $A$ et $E$ montre l'effet d'une augmentation du jeu radial relatif $\Delta$ (sans augmenter $r$ ) : cette augmentation s'accompagne d'une amélioration des caractéristiques $H / H M$ et $P / P M$ à débit partiel.

Il faut noter toutefois que dans de tels rognages, on agit aussi sur $S_{9}$ et $\alpha_{3}$ en même temps que sur $\Delta$ et $r$.

\section{Conclusions}

Un certain nombre d'autres résultats tels ceux de Rütschi [3], Salaspini [14], Evlard et Gravet [15], ou Hira et Vasandani [16], confirment le rôle essentiel joué par le bec de volute pour la détermination de la caractéristique de la machine à débit partiel. L'analyse des résultats nous indique que les paramètres les plus importants sont le rayon $r$ au bec, le jeu relatif radial $\Delta$ et la largeur relative $b_{3} / b_{2}$ :

- une augmentation du rayon au bec entraîne un accroissement des rapports $H / H M$ et une diminution des rapports $P / P M$; 


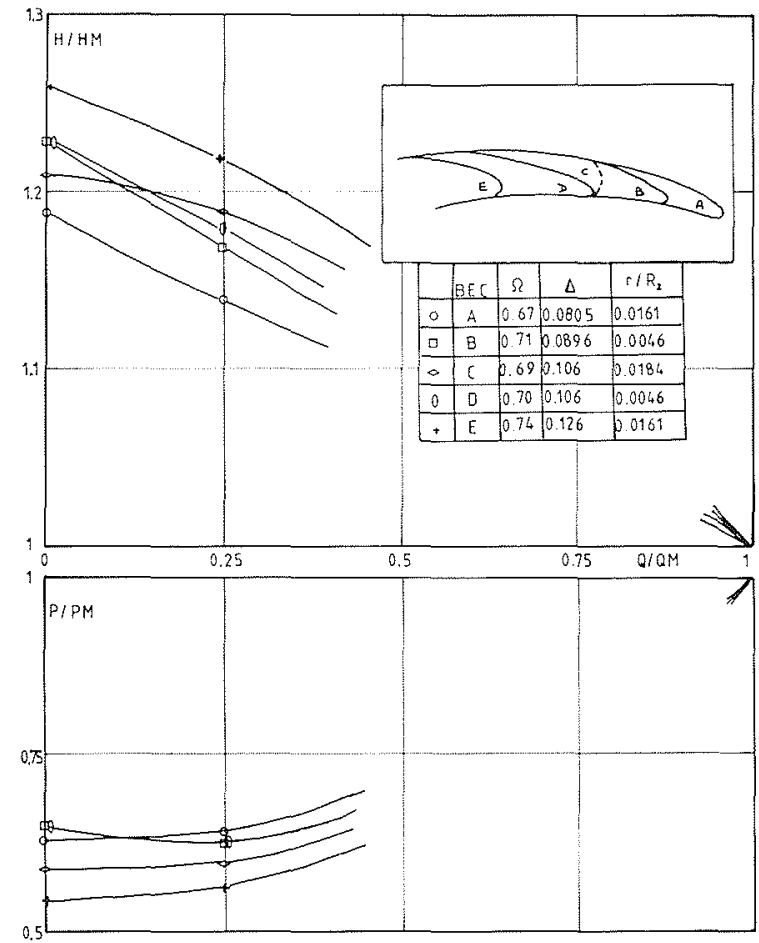

Figure 8 - Rognages d'un bec de volute.

- une augmentation du jeu relatif radial entraine une augmentation de $H / H M$ et une diminution de $P / P M$;

- une augmentation de la largeur relative $b_{3} / b_{2}$ entraîne des rapports $H / H M$ plus faibles.

Il faut rappeler cependant qu'au cours des essais évoqués, il n'y a jamais vraiment un paramètre géométrique qui varie seul. En particulier, lors d'un rognage ou d'un affutage d'un bec de volute, des paramètres tels que la section $S_{9}$ et l'angle au bec $\alpha_{3}$ évoluent en même temps que le jeu relatif $\Delta$ et le rayon au bec $r$, dans un sens qui dépend du tracé initial de la volute, et cela peut sans doute expliquer des résultats parfois contradictoires, en particulier pour les machines à petit coefficient de vitesse angulaire.

\section{Machines centrifuges à diffuseur aubé}

\section{Paramètres géométriques}

Comme dans le cas des machines à volute, les paramètres géométriques caractéristiques du couplage roue-diffuseur sont très nombreux et difficiles à faire varier indépendamment les uns des autres. Ces paramètres sont définis par la figure 9 .

Comme dans le cas précédent, nous allons passer en revue un certain nombre d'essais en notre possession pour essayer de dégager un certain nombre de tendances générales.

\section{Rapport Hydroart [17]}

Les résultats cités dans ce rapport sont analysés uniquement en ce qui concerne les rapports $H O / H M$ et $P O / P M$.

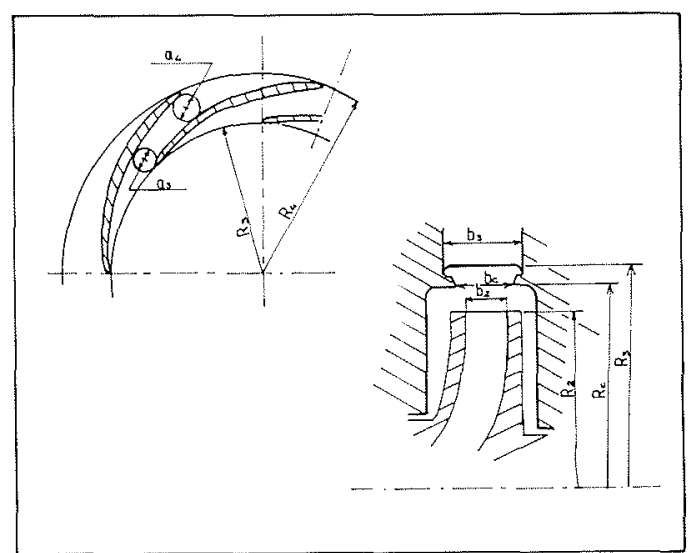

Figure 9 - Paramètres géométriques d'un diffuseur.

Le tableau I montre un certain nombre de résultats obtenus en effectuant des petites variations de $\alpha_{3}$, en maintenant constant le jeu relatif $\Delta$. De façon générale, on constate que l'augmentation de $\alpha_{3}$ se traduit par une augmentation assez nette de $H O / H M$ et très nette de PO/PM.

Tableau I
\begin{tabular}{|l|c|c|c|c|c|c|c|c|c|c|}
\hline & \multicolumn{3}{|c|}{ Essai $A$} & \multicolumn{3}{|c|}{ Essai $B$} & \multicolumn{3}{|c|}{ Essii $C$} & \multicolumn{3}{|c|}{ Essi $D$} \\
\hline$\alpha_{3}$ & $15,5^{\circ}$ & $18^{\circ}$ & $10,5^{\circ}$ & $13^{\circ}$ & $14,5^{\circ}$ & $10^{\circ}$ & $13^{\circ}$ & $11^{\circ}$ & $13^{\circ}$ & $17.5^{\circ}$ \\
\hline$H O / M M$ & 1,342 & 1,47 & 1,255 & 1,242 & 1,267 & 1,223 & 1,285 & 1,147 & 1,189 & 1,268 \\
\hline$P O / P M$ & 0.708 & 0,818 & 0.567 & 0.628 & 0,631 & 0,553 & 0,672 & 0,499 & 0,542 & 0,563 \\
\hline
\end{tabular}

Dans le tableau II sont résumés des résultats obtenus en augmentant l'entrefer roue-diffuseur par tournage du diffuseur, ce qui de toute évidence entraîne des modifications d'autres paramètres $\left(a_{3}, \alpha_{3}, r\right)$. Tous les résultats indiquent malgré tout une tendance à la diminution des rapports $H O / H M$ et $P O / P M$. A noter que l'essai $H$ est relatif à une modification au niveau du $1{ }^{\mathrm{er}}$ étage sur une machine biétagée.

Tableau II

\begin{tabular}{|l|c|c|c|c|c|c|c|c|}
\hline & \multicolumn{2}{|c|}{ Essai $E$} & \multicolumn{2}{c|}{ Essai $F$} & \multicolumn{2}{c|}{ Essai $G$} & \multicolumn{2}{c|}{ Essai $H$} \\
\hline$\Delta$ & 0,032 & 0,047 & 0,03 & 0,07 & 0,03 & 0,07 & 0,03 & 0,04 \\
\hline$H 0 / H M$ & 1,285 & 1,242 & 1,364 & 1,317 & 1,43 & 1,36 & 1,36 & 1,33 \\
\hline$P 0 / P M$ & 0,672 & 0,628 & 0,687 & 0,654 & 0,832 & 0,675 & 0,696 & 0,672 \\
\hline
\end{tabular}

Enfin, le tableau III montre qu'une augmentation de l'entrefer sur les ceintures de la roue entraîne une réduction sensible de $H O / H M$ et $P O / P M$.

Tableau III

\begin{tabular}{|c|c|c|c|c|c|}
\hline & \multicolumn{3}{|c|}{ Essai I } & \multicolumn{2}{c|}{ Essai J } \\
\hline $\begin{array}{c}\text { Entrefer } \\
\text { relatif } \\
\text { ceinture }\end{array}$ & 0,014 & 0,35 & 0,05 & 0,015 & 0,030 \\
\hline$H O / H M$ & 1,39 & 1,325 & 1,27 & 1,31 & 1,28 \\
\hline$P O / P M$ & 0,78 & 0,71 & 0,644 & 0,638 & 0,608 \\
\hline
\end{tabular}


Les essais analysés ci-dessous dans les paragraphes suivants ont été fournis par d'autres constructeurs membres du sous-groupe.

\section{Modification de $a_{3}$}

Les figures 10 et 11 montrent les modifications des caractéristiques obtenues à débit partiel lorsqu'on modifie le col $a_{3}$ du diffuseur. Les trois essais analysés indiquent que $H / H M$ et $P / P M$ ont tendance à augmenter

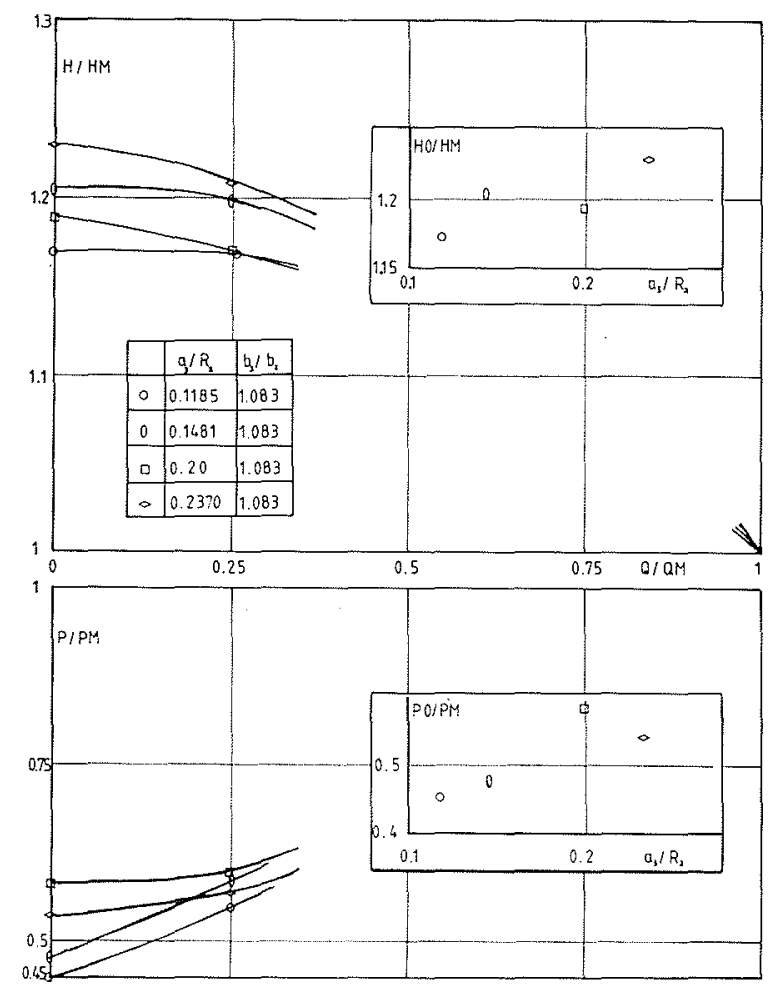

Figure 10 - Modifications de $a_{3}$.

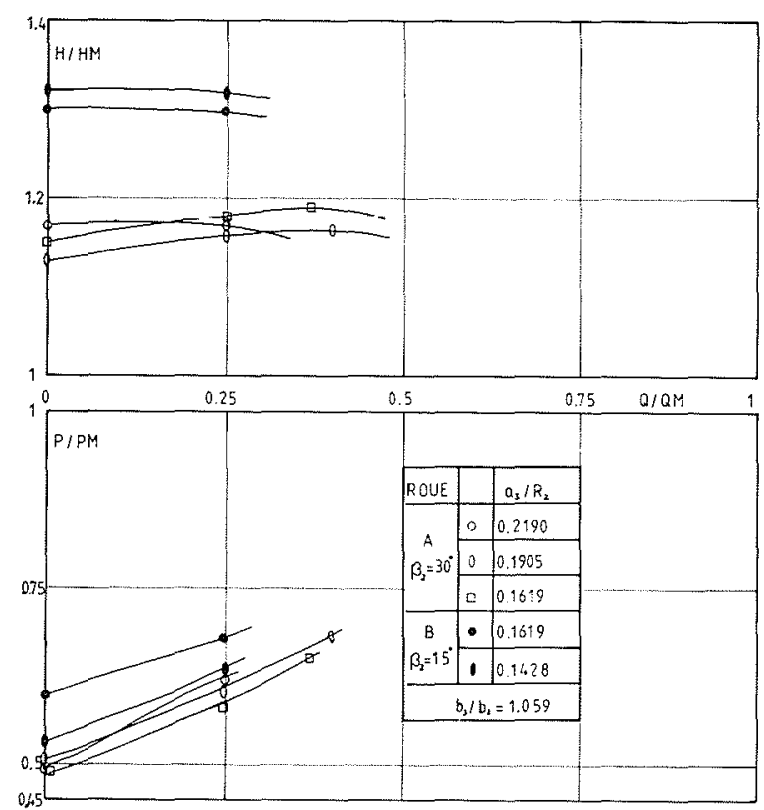

Figure 11 - Modifications de $a_{3}$. avec $a_{3} / R_{2}$. Ces résultats demeurent toutefois incomplets dans la mesure où la façon dont ont été effectuées ces modifications ne nous est pas connue pour l'instant. Compte-tenu du fait que lors de ces essais la largeur $b_{3}$ et le nombre de canaux du diffuseur ne varient pas, nous avons tracé les résultats en fonction de $a_{3} / R_{2}$ plutôt qu'en fonction du rapport $Z_{d} a_{3} b_{3} / 2 \pi R_{2} b_{2}$ qui est l'équivalent du rapport $S_{9} / 2 \pi R_{2} b_{2}$ dans les machines à simple volute.

\section{Modifications de $b_{3}$ et décalage axial du diffuseur par rapport à la roue}

Les résultats de la figure 12 permettent de noter une augmentation des rapports $H / H M$ et $P / P M$ lorsque la largeur relative augmente. De plus, on peut constater également sur cette figure l'importance de la position axiale du diffuseur par rapport à la roue : un décalage du diffuseur vers l'aspiration où le refoulement entraîne des modifications importantes des caractéristiques à débit partiel.
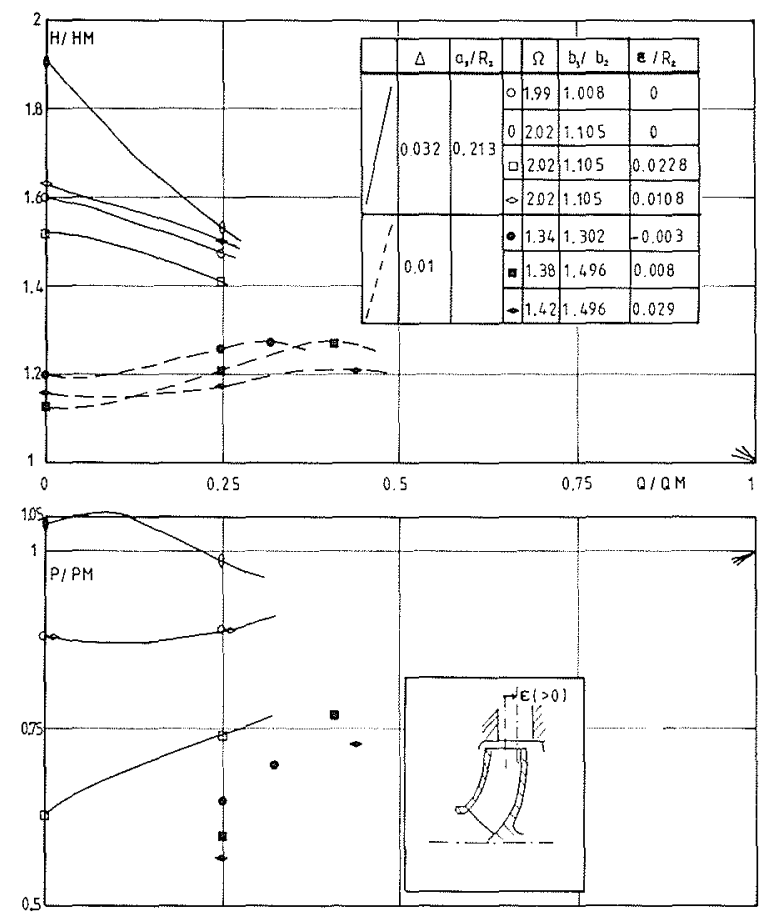

Figure 12 - Modifications de $b_{3}$ et décalage axial roue-diffuseur.

\section{Modifications diverses}

La comparaison des essais $A 1$ et $A 2, B 1$ et $B 3, C 1$ et $C 2$ (Fig. 13) donne des indications sur l'effet d'un affutage de l'entrée du diffuseur; ces affutages, qui se traduisent par une augmentation du jeu relatif, une augmentation de $r$ et $a_{3}$, de même qu'une modification de $\alpha_{3}$, entraînent une diminution de $H / H M$ à débit partiel et une augmentatin de $P / P M$. Il faut noter que le paramètre dont la variation relative est la plus grande au cours de ces essais est le rapport $r / R_{2}$, caractéristique du rayon du profil d'entrée dans le diffuseur. 
Enfin, la comparaison des essais $B 1$ et $B 2$ montre l'effet d'une modification du carter du diffuseur au voisinage de son entrée : les rapports $H / H M$ et $P / P M$ diminuent lorsqu'on augmente la largeur relative et le jeu relatif entre roue et carter.

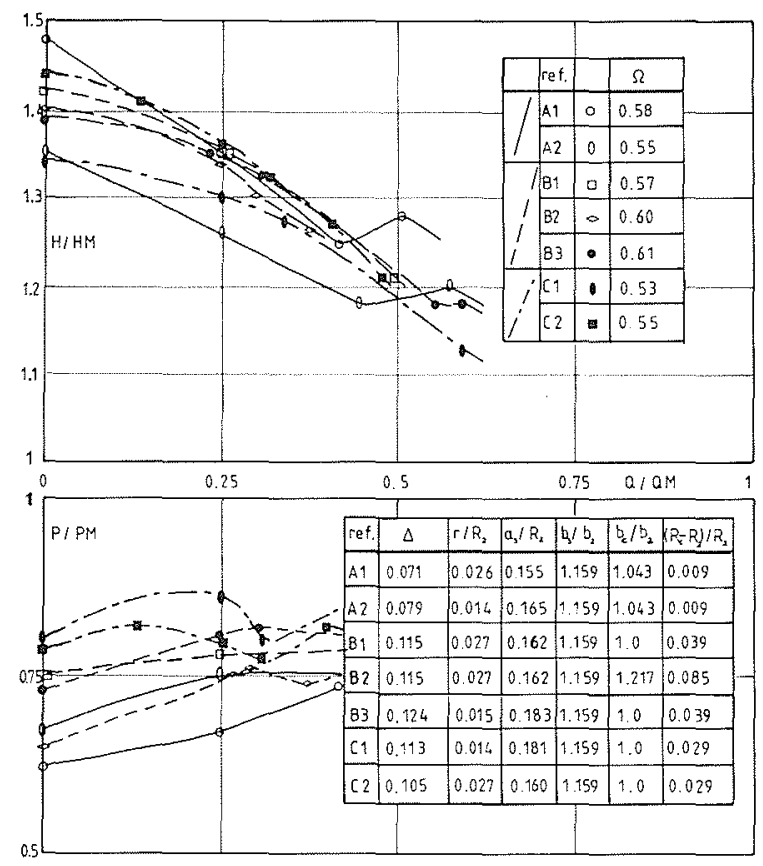

Figure 13 - Affutages divers.

\section{Conclusions}

Les essais présentés ci-dessus, de même que, par exemple, ceux de Rütschi [4], Davydov [18] ou Makay [19], montrent l'importance du couplage rouediffuseur et en particulier des paramètres géométriques suivants :

- le jeu entre la roue et le carter;

- le jeu entre la roue et l'entrée du diffuseur ;

- la section au col du diffuseur qu'on peut définir par utilisation du rapport adimensionnel $Z_{d} a_{3} b_{3} / 2 \pi R_{2} b_{2}$; - le rayon $r$ à l'entrée du diffuseur.

\section{Conclusions}

Les essais analysés ici et relatifs aux machines centrifuges à simple volute et à diffuseur aubé nous ont permis de préciser l'influence d'un certain nombre de paramètres géométriques de l'organe de récupération d'énergie cinétique sur les caractéristiques à débit partiel, bien que les paramètres envisagés n'évoluent guère indépendamment les uns des autres. Dans les volutes comme les diffuseurs, nous avons pu noter l'importance du jeu radial relatif $\Delta$, du rayon au bec de la volute ou au profil du diffuseur $r$, de la section au col $S_{9}$ ou $Z_{d} a_{3} b_{3}$ et de l'angle d'entrée $\alpha_{3}$. Enfin, au niveau des diffuseurs, nous pouvons rappeler l'influence du carter du diffuseur (jeu radial, espaces latéraux, alignement ou non du diffuseur de la roue).
D'autres essais demeurent en cours d'analyse dans le cadre de nos travaux au sein du Groupe de Travail $n^{\circ} 1$ de la SHF, en particulier un certain nombre de résultats relatifs à une même roue dans des configurations très différentes à l'aval (volute et diffuseur par exemple, ....). Nous espérons que ces divers résultats pourront apporter une contribution à la compréhension, qui reste délicate, des divers phénomènes physiques qui interviennent à débit partiel en particulier au niveau du couplage de la roue avec les parties situées à l'aval.

\section{Bibliographie}

[1] KNAPP R.T. - Centrifugal-Pump Performance as affected by design features, Transactions of the ASME, Avril 1941, p. $251-260$.

[2] RUTSCHI K. - Uber die Gestaltung stabiler Drosselkurven bei Kreisel pumpen. Schweizer Archiv. 7 Jahrgang, $n^{\circ} 8$. août 1941, p. 213-222.

[3] RUTSCHI K. - Untersuchungen an Spiralgehausepumpen verschiedener Schnelläufigkeit. Schweizer Archiv, 17 Jahrgang, $\mathrm{n}^{\circ} 2$, février 1951 , p. 33-46

[4] RUTSCHI K. - Der Einfluss der Leitvorrichtungen auf leistung und wirkungsgrad von Kreiselpumpen. Schweiz. Bauzeitung, 79, Jahrgang Helf 15, 13 avril 1961. p. 233240.

[5] CSANADY G.T. - Theory of turbomachines. Mac-Graw Hill, New-Y ork, 1964.

[6] SABERSKY R.H., ACOSTA A.J., HAUPTMANN E.G. Fluid Flow, 2nd edition. The Mac Millan Company, New York, 1971.

17] SENOO Y. - Flow in vaneless diffusers of turbomachines Separated Flows in turbomachinery components, lecture Series 1981-1, Von Karman Institute for fluid dynamics, janvier 1981, $32 \mathrm{p}$.

[8] WORSTER R.C - Further test on the flow of air through centrifugal impellers, BHRARR 508, avril $1955,14 \mathrm{p}$

[9] WORSTER R.C. - The flow in volutes and its effects on centrifugal pump performance. Proc. Instn. Mech. Engrs. vol. $177, \mathrm{n}^{\circ} 31,1963$. p. 843-875.

[10] COPLEY D.M., WORSTER R.C., ZANKER K.J. - The flow in pump volutes and its effects on performance. BHRASP 724, avril 1962, $32 \mathrm{p}$.

[11] KERMAREC, DRESSAYRE. - Influence de la géométrie des volutes sur le rendement des pompes centrifuges. Rapports partiels 1,2 et 3 du CETIM, Nantes.

[12] SUZUKI, UGAI. - Study on high specific speed airfoil fans (1st report : effects of tongue clearance and mouth ring clearance). Bull. JSME, vol. 20, $\mathrm{n}^{\circ} 143$, mai 1977 , p. $575-583$.

[13] FLORKEMEIER. - Experimentelle Untersuchungen zur optimierung von Spiralgehäusen für Kreiselpumpen mit tangentialen und radialen Druckstutzen. Dissertation, Fakultät für Maschinenbau und Elektrotechnik zu Braunschweig, 10.6.1977, 108 p.

[14] SALASPINI A. - Flows in scroll. Recent progress in pump research (10-14 décembre 1973) ; lecture séries 61 ; Von Karman Institute for fluid dynamics.

[15] EVLARD H., GRAVET S. - Pompes et groupes motopompes pour pipe-lines. $A C E C$ revue. $75 / 1-2$, p. 6-7.

[16] HIRA D.S., VASANDANI V.P. - Influence of volute tongue length and angle on the pump performance J. Inst. Engrs. (India), Mach. Eng. div., 1975, 56, 1. p. 5559.

[17] Rapport HYDROART. - Fonctionnement à débit nul des machines réversibles multi-étagées en pompe et des pompes multiétagées. 20 mars 1979.

[18] LAZARKIEWICZ S., TROSKOLANSKI A.T. - Impeller Pumps, p. 288-290. Pergamon Press, 1965.

[19] MAKAY. - Better understanding of sources of feedpump damage boosts, performance, reliability. Power, Juin 1979, p. 72-74. 


\section{Discussion}

Président : R. BRAZZINI

MM. HENRY, BORCIANI, CAIGNAERT débattent d'un problème de terminologie: "Faut-il utiliser, dans le cas général des turbomachines, les termes AMONT et AVAL, COTE HAUTE PRESSION et COTE BASSE PRESSION ou bien se référer au sens de l'écoulement ?" M. BORCIANI signale en particulier que le problème a été résolu par le IEC/TC4 d'une façon générale en appelant un côté High Pressure Side et l'autre Low Pressure Side.

A la suite des interventions de MM. OSTERWALDER et
BARRAND, M. CAIGNAERT précise qu'il n'existe quasiment aucun travail, ni aucune statistique sur le sujet qu'il a abordé. De plus, il est impossible de faire une analyse détaillée des pertes dans les machines faute d'informations sur leur géométrie et de résultats d'essais suffisamment complets.

M. ANDRE signale que des essais systématiques ont été effectués chez le constructeur de ventilateur BERRY, pour étudier l'influence du bec de volute sur les courbes de rendement.

\section{Abstract \\ Influence of the geometry of the downstream section of the impeller on the characteristics of partial flow pumps}

This paper discusses the influence of geometrical parameters of vaned diffusors and volutes on partial-flow characteristics of centrifugal pumps. The analysed results originate from publications or some manufacturers' internal notes, members of the 1-c working group of the "Sociéte Hydro" technique de France". Some test results involving only modifications of the casing downstream of the impeller are presented here in a non-dimensional form.

Generally speaking, these modifications concern many geometrical parameters, and it is not easy to characterise the results: only trends emerge. These trends vary according to the type of pump (specific speed influence) and to the impeller geometry: general trend seems to be much more difficult to obtain for pumps operating at low specific speed. The first part shows the influence of some changes made in the volute on the partial-flow characteristics of singlevolute pumps. The volute tongue appears to be most important, especially the relative radial gap and the tongue radius: head coefficients increase and power co-efficients tend to decrease when these parameters increase.

The second part covers the results correlated to modifications of vaned-diffusor inlet parts. The influence of the relative radial gap, the blade inlet radius, the blade inlet angle, the diffusor throat area, the diffusor's axial position in relation to the impeller and gaps between impeller and-casing can be revealed in the same way. 